\title{
Multi-Wavelength Observations of the Radio Magnetar PSR J1622-4950 and Discovery of its Possibly Associated Supernova Remnant
}

\section{Citation}

Anderson, Gemma E., B. M. Gaensler, Patrick O. Slane, Nanda Rea, David L. Kaplan, Bettina Posselt, Lina Levin, et al. 2012. "MULTI-WAVELENGTH OBSERVATIONS OF THE RADIO MAGNETAR PSR J1622-4950 AND DISCOVERY OF ITS POSSIBLY ASSOCIATED SUPERNOVA REMNANT." The Astrophysical Journal 751 (1) (May 4): 53. doi:10.1088/0004-637x/751/1/53.

\section{Published Version}

doi:10.1088/0004-637x/751/1/53

\section{Permanent link}

http://nrs.harvard.edu/urn-3:HUL.InstRepos:14068407

\section{Terms of Use}

This article was downloaded from Harvard University's DASH repository, and is made available under the terms and conditions applicable to Other Posted Material, as set forth at http:// nrs.harvard.edu/urn-3:HUL.InstRepos:dash.current.terms-of-use\#LAA

\section{Share Your Story}

The Harvard community has made this article openly available.

Please share how this access benefits you. Submit a story.

Accessibility 


\title{
MULTI-WAVELENGTH OBSERVATIONS OF THE RADIO MAGNETAR PSR J1622-4950 AND DISCOVERY OF ITS POSSIBLY ASSOCIATED SUPERNOVA REMNANT
}

\author{
Gemma E. Anderson ${ }^{1}$, B. M. Gaensler ${ }^{1}$, Patrick O. Slane ${ }^{2}$, Nanda Rea ${ }^{3}$, David L. Kaplan ${ }^{4}$, Bettina Posselt ${ }^{5}$, \\ Lina Levin $^{6,7}$, Simon Johnston ${ }^{7}$, Stephen S. Murray ${ }^{8}$, Crystal L. Brogan ${ }^{9}$, Matthew Bailes ${ }^{6}$, Samuel Bates $^{10,11}$, \\ Robert A. Benjamin ${ }^{12}$, N. D. Ramesh Bhat ${ }^{6}$, Marta Burgay ${ }^{13}$, Sarah Burke-Spolaor ${ }^{7,14}$, Deepto Chakrabarty $^{15}$, \\ Nichi D’Amico ${ }^{13}$, Jeremy J. Drake ${ }^{2}$, Paolo Esposito ${ }^{13}$, Jonathan E. Grindlay ${ }^{2}$, Jaesub Hong ${ }^{2}$, G. L. Israel ${ }^{16}$, \\ Michael J. Keith ${ }^{7}$, Michael Kramer ${ }^{10,17}$, T. Joseph W. Lazio ${ }^{14}$, Julia C. LeE ${ }^{2}$, Jon C. Mauerhan ${ }^{18}$, Sabrina Milia ${ }^{13,19}$, \\ Andrea Possenti ${ }^{13}$, Ben Stappers ${ }^{10}$, and Danny T. H. Steeghs ${ }^{20}$ \\ ${ }^{1}$ Sydney Institute for Astronomy, School of Physics A29, The University of Sydney, NSW 2006, Australia; g.anderson@physics.usyd.edu.au \\ ${ }^{2}$ Harvard-Smithsonian Center for Astrophysics, Cambridge, MA 02138, USA \\ ${ }^{3}$ Institut de Ciencies de 1'Espai (CSIC-IEEC), Campus UAB, Facultat de Ciencies, Torre C5-parell, 2a planta, 08193, Bellaterra, Barcelona, Spain \\ ${ }^{4}$ Department of Physics, University of Wisconsin, Milwaukee, WI 53201, USA \\ ${ }^{5}$ Department of Astronomy and Astrophysics, Pennsylvania State University, PA 16802, USA \\ ${ }^{6}$ Centre for Astrophysics and Supercomputing, Swinburne University of Technology, VIC 3122, Australia \\ ${ }^{7}$ Australia Telescope National Facility, CSIRO Astronomy and Space Science, P.O. Box 76, Epping, NSW 1710, Australia \\ ${ }^{8}$ Department of Physics and Astronomy, John Hopkins University, Baltimore, MD 21218, USA \\ ${ }^{9}$ National Radio Astronomy Observatory, Charlottesville, VA 22903, USA \\ 10 Jodrell Bank Centre for Astrophysics, School of Physics and Astronomy, University of Manchester, Manchester M13 9PL, UK \\ ${ }_{11}^{11}$ Physics Department, West Virginia University, Morgantown, WV 2650, USA \\ ${ }^{12}$ Department of Physics, University of Wisconsin, Whitewater, WI 53190, USA \\ ${ }^{13}$ INAF/Osservatorio Astronomico di Cagliari, 09012 Capoterra, Italy \\ ${ }^{14}$ Jet Propulsion Laboratory, California Institute of Technology, Pasadena, CA 91109, USA \\ ${ }^{15}$ MIT Kavli Institute for Astrophysics and Space Research and Department of Physics, Massachusetts Institute of Technology, Cambridge, MA 02139, USA \\ ${ }^{16} \mathrm{INAF} /$ Osservatorio Astronomico di Roma, Monteporsio Catone, Italy \\ ${ }^{17}$ Max Planck Institut für Radioastronomie, 53121 Bonn, Germany \\ ${ }^{18}$ Spitzer Science Center, California Institute of Technology, Pasadena, CA 91125, USA \\ ${ }^{19}$ Dipartimento di Fisica, Universita' degli Studi di Cagliari, Cittadella Universitaria, 09042 Monserrato (CA), Italy \\ ${ }^{20}$ Department of Physics, University of Warwick, Coventry CV4 7AL, UK \\ Received 2011 September 15; accepted 2012 March 9; published 2012 May 4
}

\begin{abstract}
We present multi-wavelength observations of the radio magnetar PSR J1622-4950 and its environment. Observations of PSR J1622-4950 with Chandra (in 2007 and 2009) and XMM (in 2011) show that the X-ray flux of PSR J1622-4950 has decreased by a factor of $\sim 50$ over 3.7 years, decaying exponentially with a characteristic time of $\tau=360 \pm 11$ days. This behavior identifies PSR J1622-4950 as a possible addition to the small class of transient magnetars. The X-ray decay likely indicates that PSR J1622-4950 is recovering from an X-ray outburst that occurred earlier in 2007, before the 2007 Chandra observations. Observations with the Australia Telescope Compact Array show strong radio variability, including a possible radio flaring event at least one and a half years after the 2007 X-ray outburst that may be a direct result of this X-ray event. Radio observations with the Molonglo Observatory Synthesis Telescope reveal that PSR J1622-4950 is $8^{\prime}$ southeast of a diffuse radio arc, G333.9+0.0, which appears non-thermal in nature and which could possibly be a previously undiscovered supernova remnant (SNR). If G333.9+0.0 is an SNR then the estimates of its size and age, combined with the close proximity and reasonable implied velocity of PSR J1622-4950, suggest that these two objects could be physically associated.
\end{abstract}

Key words: ISM: individual objects (G333.9+0.0) - ISM: supernova remnants - pulsars: individual (PSR J1622-4950) - radio continuum: stars - stars: neutron - X-rays: stars

Online-only material: color figures

\section{INTRODUCTION}

"Magnetar" has become the commonly used term to describe the emerging class of rare, young, and highly magnetized neutron stars $\left(B \gtrsim 10^{14} \mathrm{G}\right)$ referred to as anomalous X-ray pulsars and soft gamma repeaters (SGRs), with perhaps as many as 23 now detected. ${ }^{21}$ Magnetars are primarily bright X-ray emitters, for which most of this high energy radiation is thought to be generated through the decay of their strong magnetic fields (for a review on magnetars see Mereghetti 2008). While magnetars may be X-ray luminous, only three such sources have been detected at radio wavelengths: XTE J1810-197 (Halpern

\footnotetext{
21 http://www.physics.mcgill.ca/ pulsar/magnetar/main.html
}

et al. 2005; Camilo et al. 2006), 1E 1547.0-5408 (Camilo et al. 2007b), and most recently PSR J1622-4950 (Levin et al. 2010).

PSR J1622-4950 is unusual as it is the first magnetar to have been discovered by its pulsed radio emission (Levin et al. 2010). PSR J1622-4950 was detected as a $4.3 \mathrm{~s}$ period radio pulsar in the High Time Resolution Universe survey performed with the Parkes $64 \mathrm{~m}$ telescope (Keith et al. 2010) and was then recovered in other archival radio data sets (Levin et al. 2010). Levin et al. (2010) showed this pulsar to be very different from ordinary radio pulsars in that it displays large variations in flux over time, with time spans of inactivity during which it is undetected. Its variable pulse profile and high inferred magnetic field strength show that it has similar properties to the only other two known radio magnetars, XTE J1810-197 and 1E 1547.0-5408. Both of 
Table 1

X-Ray Observations of PSR J1622-4950

\begin{tabular}{|c|c|c|c|c|c|c|}
\hline Telescope & $\begin{array}{c}\text { ObsID } \\
\text { Instrument }\end{array}$ & $\begin{array}{c}\text { Date }^{\mathrm{a}} \\
\text { Exp Time }(\mathrm{ks})^{\mathrm{b}}\end{array}$ & $\mathrm{MJD}^{\mathrm{c}}$ & $\begin{array}{l}\text { Count-rate } \\
(\text { counts ks } \\
\left.\text { ch }^{-1}\right)\end{array}$ & $\begin{array}{l}\text { ACIS-S rate } \\
(\text { counts ks } \\
-1\end{array}$ & $\begin{array}{l}\text { Off-axis Angle } \\
\text { (arcmin) }\end{array}$ \\
\hline Chandra & $\begin{array}{c}8161 \\
\text { ACIS-S }\end{array}$ & $\begin{array}{c}2007-06-13 \\
2.02\end{array}$ & 54264 & $96.8 \pm 6.9$ & 96.8 & 4.0 \\
\hline Chandra & $\begin{array}{c}9911 \\
\text { ACIS-I }\end{array}$ & $\begin{array}{c}2009-06-14 \\
60.10\end{array}$ & 54996 & $9.7 \pm 0.6$ & 10.8 & 16.1 \\
\hline Chandra & $\begin{array}{c}10929 \\
\text { ACIS-I }\end{array}$ & $\begin{array}{c}2009-07-10 \\
19.90\end{array}$ & 55022 & $7.1 \pm 0.6$ & 9.3 & 1.8 \\
\hline$X M M$ & $\begin{array}{c}0654110101 \\
\text { EPIC-PN }\end{array}$ & $\begin{array}{c}2011-02-22 \\
46.4\end{array}$ & 55615 & $5.8 \pm 0.6$ & 1.8 & 1.1 \\
\hline
\end{tabular}

Notes.

a The date is in the form yyyy-mm-dd.

b The quoted exposure times are the effective exposure time after time intervals when there was flaring or when

the source was on the chip gap have been removed.

${ }^{c}$ Modified Julian Date

d Total observed count rate in the $0.3-8.0 \mathrm{keV}$ energy range.

e Model predicted ACIS-S equivalent count rate when compared to the 2007 June Chandra observation in the $0.3-8.0 \mathrm{keV}$ energy range.

these radio magnetars also fit into an emerging subgroup known as transient magnetars, which randomly undergo bright X-ray bursts with increases in X-ray flux up to a factor of several hundred (for example see Tam et al. 2006; Bernardini et al. 2009, 2011; Scholz \& Kaspi 2011). It appears that for both XTE J1810-197 and 1E 1547.0-5408, the pulsed radio emission turned on as a result of an X-ray outburst.

In this paper, we present multi-wavelength data that further confirm the magnetar identification of PSR J1622-4950 and that support its transient nature. New and archival radio, infrared and X-ray observations are presented in Section 2 and the corresponding results are presented in Section 3. In Section 4, we use these data to explore PSR J1622-4950's X-ray and radio variability over the last 10 years, and to study the variable nature of its polarized radio emission. We also discuss a possible $\gamma$-ray counterpart to PSR J1622-4950 and identify a possibly associated young supernova remnant (SNR).

\section{OBSERVATIONS}

\subsection{X-Ray Observations}

\subsubsection{Chandra Observations}

The position of PSR J1622-4950 was observed three times with the Chandra X-ray Observatory, using the Advanced CCD Imaging Spectrometer (ACIS; Garmire et al. 2003). The first observation took place on 2007 June 13 as part of the "ChIcAGO" (Chasing the Identification of ASCA Galactic Objects) project. ChIcAGO is a survey designed to localize and classify the unidentified X-ray sources discovered during the ASCA Galactic Plane Survey (AGPS; Sugizaki et al. 2001; see Anderson et al. 2011 for some of the first ChIcAGO results; further details will be published by G. E. Anderson et al., in preparation.) PSR J1622-4950 was observed again on 2009 June 14 and on 2009 July 10 . These data were reduced using the Chandra Interactive Analysis of Observations (CIAO) software, version 4.3, following the online CIAO 4.3 Science Threads. ${ }^{22}$ For details on these Chandra observations see Table 1.

\footnotetext{
22 http://cxc.harvard.edu/ciao/threads/
}

\subsubsection{XMM-Newton Observations}

We observed PSR J1622-4950 with XMM-Newton starting on 2011 February 22. The PN (Strüder et al. 2001) and MOS (Turner et al. 2001) cameras, which comprise the European Photon Imaging Camera (EPIC), were operated in Full Frame mode. Data were processed using SAS version 10.0.0, ${ }^{23}$ and we employed the latest calibration files available at the time the reduction was performed (2011 March). Standard datascreening criteria were applied in the extraction of scientific products. The observation was highly affected by proton flares, which we had to cut from our data before proceeding with the scientific analysis, resulting in a net livetime exposure of $46.4 \mathrm{ks}$. Since both MOS and PN give consistent results, in the following we report only on the high-time-resolution PN data. Further details on the $X M M / E P I C-P N$ observation can be found in Table 1.

\subsection{Infrared and Optical Observations}

We compared the Chandra position of PSR J1622-4950 (see Section 3.1.1. below) to optical and infrared point source catalogs and survey images including the U.S. Naval Observatory B catalog, version 1.0 (Monet et al. 2003), the Two Micron All Sky Survey (2MASS; Skrutskie et al. 2006), the Galactic Legacy Infrared Mid-Plane Survey Extraordinaire (GLIMPSE; Benjamin et al. 2003), and the $24 \mu \mathrm{m}$ images from the MIPSGAL Survey (Carey et al. 2009), but no counterpart to PSR J1622-4950 was identified in any of these data sets. On 2007 June 24 we observed the position of PSR J1622-4950 in the $K_{s}$ band for 13.5 minutes using the Persson's Auxiliary Nasmyth Infrared Camera (PANIC: Martini et al. 2004; Osip et al. 2008) on the $6.5 \mathrm{~m}$ Magellan I (Baade) telescope, located at Las Campanas Observatory. The final image was a combination of $30 \mathrm{~s}$ exposures carried out three times at each position of a nine-point dither pattern to account for the high background. Standard reduction procedures were applied using the IRAF software package (Tody 1986, 1993). We calibrated the field's photometry and astrometry using the source extraction software

23 http://xmm.esac.esa.int/sas/ 
Table 2

ATCA Observations of PSR J1622-4950

\begin{tabular}{|c|c|c|c|c|c|c|c|}
\hline \multirow{2}{*}{$\begin{array}{l}\text { Date } \\
\text { (yyyy-mm-dd) }\end{array}$} & \multirow[t]{2}{*}{ MJD } & \multirow{2}{*}{$\begin{array}{c}\text { Central Frequency } \\
(\mathrm{MHz})\end{array}$} & \multirow{2}{*}{$\begin{array}{l}\text { Flux Density } \\
\text { (mJy) }\end{array}$} & \multirow{2}{*}{$\begin{array}{c}\text { Spectral Index } \\
(\alpha)\end{array}$} & \multicolumn{3}{|c|}{ Polarization } \\
\hline & & & & & $\begin{array}{c}\text { Linear } \\
(\mathrm{mJy})\end{array}$ & $\begin{array}{l}\text { P.A. } \\
\text { (deg) }\end{array}$ & $\begin{array}{c}\text { Circular } \\
\text { (mJy) }\end{array}$ \\
\hline \multirow[t]{2}{*}{$2008-11-22$} & 54793 & 5312 & $33.0 \pm 0.3$ & $-0.13 \pm 0.04$ & $26.6 \pm 0.7(79 \%)$ & $-17.5 \pm 0.5$ & $\lesssim 2.0(\lesssim 6 \%)$ \\
\hline & & 8768 & $30.9 \pm 0.6$ & & $25.0 \pm 0.8(81 \%)$ & $-25.8 \pm 0.7$ & $\lesssim 2.5(\lesssim 8 \%)$ \\
\hline \multirow[t]{2}{*}{$2008-12-05$} & 54806 & 4800 & $40.4 \pm 0.3$ & $-0.44 \pm 0.04$ & $5.7 \pm 0.4(14 \%)$ & $+26.7 \pm 1.5$ & $-6.2 \pm 0.3(15 \%)$ \\
\hline & & 8256 & $31.9 \pm 0.6$ & & $5.8 \pm 0.7(18 \%)$ & $-22.5 \pm 2.5$ & $-4.8 \pm 0.5(15 \%)$ \\
\hline 2009-12-08 & 55174 & 5500 & $13 \pm 1$ & $+0.2 \pm 0.2$ & & & \\
\hline $2010-02-27^{a}$ & 55255 & 9000 & $14.3 \pm 0.8$ & & & & \\
\hline
\end{tabular}

Notes. All errors are 1 $\sigma$. The circular polarization upper bounds for the 2008-11-22 observations are limits on the magnitude.

a The flux densities quoted are the average values at 5.5 and $9 \mathrm{GHz}$ from the 2009-12-08 and 2010-02-27 ATCA observations, taken from Levin et al. (2010).

SExtractor (Bertin \& Arnouts 1996) with comparisons to the 2MASS point source catalog (2MASS PSC), which has a position uncertainty of 0.1 (Skrutskie et al. 2006). No infrared counterpart was detected within 0 ". 8 of the X-ray position of PSR J1622-4950 to a lower limit $K_{s} \geqslant 20.7$.

\subsection{Radio Observations}

\subsubsection{Archival}

PSR J1622-4950 is coincident with a bright knot of emission that is part of a larger diffuse radio source seen in the first and second epoch Molonglo Galactic Plane Surveys (MGPS1 and MGPS2, respectively; Green et al. 1999; Murphy et al. 2007) and also seen, albeit less clearly, in the continuum maps from the Southern Galactic Plane Survey (SGPS: Haverkorn et al. 2006). This feature will be discussed in Section 3.2.1. The MGPS surveys were performed at $843 \mathrm{MHz}$ with the Molonglo Observatory Synthesis Telescope (MOST) at a resolution of $43^{\prime \prime}$ while the continuum SGPS images were created from Australia Telescope Compact Array (ATCA) $1.4 \mathrm{GHz}$ observations at a resolution of $100^{\prime \prime}$.

\subsubsection{ATCA Observations}

We observed PSR J1622-4950 with ATCA simultaneously at both 5 and $9 \mathrm{GHz}$, with a $128 \mathrm{MHz}$ bandwidth in each band, on 2008 November 22 and 2008 December 5 as summarized in Table 2. A mosaic pattern was used in an attempt to both detect the magnetar and study the morphology of the surrounding diffuse emission seen in the MGPS1, MGPS2, and SGPS. Both frequencies were observed for 6.79 and $8.01 \mathrm{hr}$ in the EW367 and 750B configurations, respectively, using PKS B1934-638 for flux calibration and PMN J1603-4904 for phase and polarization calibration. Data reduction and analysis were performed using the MIRIAD ${ }^{24}$ software package using standard techniques.

\section{RESULTS}

\subsection{X-Ray Results}

\subsubsection{X-Ray Counterpart to PSR J1622-4950}

The 2007 June Chandra observation was an attempt to localize the unidentified AGPS source AX J162246-4946 as part of the ChIcAGO survey. Six point sources were detected within $4^{\prime}$ of the position of AX J162246-4946, all of which

\footnotetext{
24 http://www.atnf.csiro.au/computing/software/miriad/
}

may have contributed to the X-ray emission originally detected with ASCA on 1999 April 22 as part of the AGPS. The brightest source, CXOU J162244.8-495054 (Evans et al. 2010), which Levin et al. (2010) identified as the X-ray counterpart to PSR J1622-4950, was at least 20 times brighter than the other five sources. As CXOU J162244.8-495054 fell in the gap between CCDs for part of the 2007 June observation we only included those time intervals where the source was detected, resulting in an effective exposure time of $\sim 2.02 \mathrm{ks}$ and a count rate of $96.8 \pm 6.9$ counts $\mathrm{ks}^{-1}$ in the $0.3-8.0 \mathrm{keV}$ energy range.

The 2009 July Chandra observation, two years later, also detected the same six sources in the $4^{\prime}$ region surrounding the position of AX J162246-4946. While the 2009 July observation was $\sim 10$ times longer than the 2007 June observation, CXOU J162244.8-495054 had faded significantly with a much lower count rate of $7.1 \pm 0.6$ counts $\mathrm{ks}^{-1}$ (equivalent to $\sim 9.3$ counts $\mathrm{ks}^{-1}$ when compared to the 2007 June observation in the ACIS-S configuration ${ }^{25}$ ) in the $0.3-8.0 \mathrm{keV}$ energy range. The X-ray flux of CXOU J162244.8-495054 had therefore reduced by a factor of $\sim 10$ in the two years between the 2007 June and 2009 July Chandra observations. The best X-ray position for CXOU J162244.8-495054, to within a 0.8 radius circle for a $95 \%$ confidence $^{26}$ is (J2000) R.A. = 16:22:44.89 and decl. $=-49: 50: 52.7$ taken from the 2009 July Chandra observation. This agrees within $95 \%$ confidence of the X-ray position given by Levin et al. (2010). From now on we will refer to both the X-ray counterpart, CXOU J162244.8-495054, and radio counterpart of this magnetar as PSR J1622-4950.

The Chandra observation from approximately one month earlier (2009 June) also detected PSR J1622-4950 very far offaxis, showing a count rate slightly higher than that of the 2009 July observation. This is equivalent to $\sim 10.8$ counts $\mathrm{ks}^{-1}$ in the $0.3-8.0 \mathrm{keV}$ energy range when compared to the 2007 June Chandra observation. In 2011 February, our XMM observation showed that PSR J1622-4950 had faded even more, resulting in an EPIC-PN count rate of $5.8 \pm 0.6$ count $\mathrm{ks}^{-1}$ in the $0.4-10.0 \mathrm{keV}$ energy range (equivalent to $\sim 1.8$ counts $\mathrm{ks}^{-1}$ in the ACIS-S configuration of 2007 June in the $0.3-8.0 \mathrm{keV}$ energy range). A summary of each X-ray observation can be found in Table 1.

\footnotetext{
25 All count rate conversions are based on the X-ray spectral fit of the 2007 June Chandra observation, derived in Section 3.1.2, in order to account for the differences in the off-axis position and between the X-ray telescope instruments.

26 This error takes into account the Chandra absolute positional accuracy (see http://cxc.harvard.edu/cal/ASPECT/celmon/) and the position uncertainties associated with the CIAO source detection algorithm wavdetect (Hong et al. 2005).
} 
It is also worth exploring to what extent the X-ray emission from PSR J1622-4950 may have contributed to the flux from AX J162246-4946 in the 1999 ASCA observation. PSR J1622-4950 lies $4^{\prime}$ away from the position of AX J162246-4946 (Sugizaki et al. 2001). The other five point sources that were detected in both the 2007 June and 2009 July Chandra observations all fall within $3^{\prime}$ of the position of AX J162246-4946. By fitting an absorbed power law to the spectrum of each of these point sources, assuming a power-law spectral index of 2 and $N_{\mathrm{H}}=2 \times 10^{22} \mathrm{~cm}^{-2}$, we estimate that they contributed a total absorbed flux of $\sim 1 \times 10^{-13} \mathrm{erg} \mathrm{cm}^{-2} \mathrm{~s}^{-1}$ in the $0.3-10.0 \mathrm{keV}$ energy range. (The fluxes of three out of the five point sources were unchanged within a factor of two between the 2007 June and 2009 July Chandra observations. The flux of the fourth point sources may have decreased by a factor of $\sim 4$ between 2007 and 2009 and the flux of the fifth point source may have increased by a factor of $\sim 6$.) Using the 2.0-10.0 keV ASCA count rates (Sugizaki et al. 2001) and the absorbed power-law spectral fit described above, we estimate an absorbed X-ray flux of $\sim 4 \times 10^{-13} \mathrm{erg} \mathrm{cm}^{-2} \mathrm{~s}^{-1}$ from AX J162246-4946. Subtracting the contribution from the five nearby point sources described above, we estimate that the absorbed X-ray flux from PSR J1622-4950 contributed, at most, $\sim 3 \times 10^{-13} \mathrm{erg} \mathrm{cm}^{-2} \mathrm{~s}^{-1}$ (corresponding to $75 \%$ ) to the observed X-ray emission seen from AX J162246-4946 in the AGPS.

\subsubsection{X-Ray Spectrum and Variability}

In all three Chandra observations we extracted the X-ray spectrum of PSR J1622-4950 using the CIAO tool specextract. As the source fell close to the edge of the CCD in the 2007 June observation and was very far off-axis in the 2009 June observation, specextract was run in extended source mode to better handle the creation of response files. The spectra from the 2007 June and 2009 July Chandra observations were binned before fitting to include at least 10 counts bin $^{-1}$, as this is the minimum number of counts required to result in a statistically significant fit. The small number of counts detected, combined with the large absorption in the Galactic plane, resulted in very few photons being detected below $2 \mathrm{keV}$ in these two Chandra observations. A larger number of counts were detected during the 2009 June Chandra observation (the source had faded but the observation was long with the source off-axis) so we chose to bin its spectrum to include at least 40 counts bin $^{-1}$. The $X M M /$ EPIC-PN source events and spectrum were extracted within a circular region of $20^{\prime \prime}$ centered on the peak of the point-spread function of the source, while the background was obtained from a source-free region of similar size. When generating the EPIC-PN spectrum we have included events with PATTERN $\leqslant 4$ (i.e., single and double events). This $X M M$ spectrum was binned before fitting, using at least 40 counts bin $^{-1}$ to compensate for the high background and not oversampling the intrinsic spectral resolution by more than a factor of three. ${ }^{27}$

The X-ray spectra of magnetars are commonly fit using a blackbody-plus-power-law model or a multiple blackbody model. We chose to simultaneously fit the four X-ray observations of PSR J1622-4950 with both a single absorbed blackbody model and a single absorbed power-law model as the low number of counts preclude the identification of multiple components. The spectra were fit using XSPEC (Dorman \& Arnaud

\footnotetext{
27 http://xmm.esa.int/sas/10.0.0/doc/specgroup/node14.html
}

2001) with the absorption, $N_{\mathrm{H}}$, set as a free parameter but constrained to have the same value at each epoch, while the other parameters were set to vary individually. It should be noted that the resulting spectrum and fit to the June 2007 Chandra observation of PSR J1622-4950 may not be entirely accurate given its location close to the edge of the CCD, which resulted in the source dithering off the chip at regular intervals. By removing those time intervals where the source was not on the chip we have reduced this error but there can still be problems associated with the response files created during the extraction process. ${ }^{28}$

A simultaneous spectral fit with either an absorbed blackbody or an absorbed power-law model are equally reasonable for describing the four X-ray spectra of PSR J1622-4950, both resulting in $\chi_{\text {red }}^{2}=0.7$. The blackbody and power-law spectral fits resulted in absorptions of $N_{\mathrm{H}}=5.4_{-1.4}^{+1.6} \times 10^{22} \mathrm{~cm}^{-2}$ and $N_{\mathrm{H}}=10.5_{-2.1}^{+2.5} \times 10^{22} \mathrm{~cm}^{-2}$ (errors at $90 \%$ confidence), respectively, using abundances from Lodders (2003) and the photoelectric scattering section from Balucinska-Church \& McCammon (1992) and Yan et al. (1998). These absorptions exceed the Galactic $N_{\mathrm{H}}$ at the position of PSR J1622-4950 predicted from Hi surveys (Dickey \& Lockman 1990; Kalberla et al. 2005) by a factor of two to four, as expected at high column densities and low Galactic latitudes (e.g., Arabadjis \& Bregman 1999). The absorbed blackbody and absorbed powerlaw fit parameters for each spectrum are listed in Table 3.

In order to investigate the long-term X-ray variability of PSR J1622-4950 we computed the absorbed and unabsorbed fluxes and their uncertainties for the four X-ray spectra, using the XSPEC model cflux, in the $0.3-10.0 \mathrm{keV}$ energy range. These fluxes are listed in Table 3. (As the absorbed X-ray fluxes calculated from the blackbody and power-law spectral fits are the same at each epoch within the $90 \%$ confidence we will use the fluxes obtained from the blackbody fit in the rest of our analysis unless otherwise stated. This investigation was also limited to the absorbed fluxes as the errors on the unabsorbed power-law fluxes are very large and unconstraining.) The 2007 June Chandra observation is our brightest X-ray detection of PSR J1622-4950, making it 9 times brighter than the two mid-2009 Chandra observations and 47 times brighter than in the 2011 February $X M M$ observation. As described in Section 3.1.1, PSR J1622-4950 has an X-ray flux upper limit of $F_{x} \leqslant 3 \times 10^{-13} \mathrm{erg} \mathrm{cm}^{-2} \mathrm{~s}^{-1}(0.3-10.0 \mathrm{keV})$ in the 1999 April $A S C A$ observation. This flux upper limit is $\gtrsim 6$ times fainter than our X-ray flux measurement from the 2007 June Chandra observation but is consistent with the fluxes measured at the other three X-ray epochs.

The best-fit blackbody temperatures $(k T)$ and power-law spectral indices $(\Gamma)$ of the simultaneous spectral fits are also listed in Table 3. While both of these parameters at each epoch are on the high end when compared to those seen from other known magnetars, ${ }^{29}$ they are not unreasonable (for example, see Gelfand \& Gaensler 2007; Enoto et al. 2009). The parameters $k T$ and $\Gamma$ also indicate there was no significant spectral variability as these values remain the same between epochs within the $90 \%$ confidence. The spectra with the absorbed blackbodies are plotted in Figure 1.

We further investigated evidence of spectral evolution by comparing the hardness ratios resulting from simultaneously fitting all four spectra with an absorbed blackbody model and

\footnotetext{
28 For caveats associated with the analysis of sources near the edge of a CCD see http://cxc.harvard.edu/ciao/ahelp/specextract.html.

29 See http://www.physics.mcgill.ca/ pulsar/magnetar/main.html.
} 
Table 3

X-Ray Spectral Modeling of PSR J1622-4950

\begin{tabular}{|c|c|c|c|c|c|c|}
\hline \multirow{2}{*}{$\begin{array}{l}\text { Telescope } \\
\text { Date }\end{array}$} & \multicolumn{3}{|c|}{ Absorbed Blackbody Fit ${ }^{\mathrm{a}}$} & \multicolumn{3}{|c|}{ Absorbed Power-law Fit ${ }^{\mathrm{a}}$} \\
\hline & $\begin{array}{l}k T \\
N_{\mathrm{H}}\end{array}$ & $\begin{array}{c}F_{x, \text { abs }} \\
\chi_{\text {red }}^{2} \\
\end{array}$ & $\begin{array}{l}F_{x, \text { unab }} \\
L_{x, \text { unab }}\end{array}$ & $\begin{array}{c}\Gamma \\
N_{\mathrm{H}} \\
\end{array}$ & $\begin{array}{c}F_{x, \text { abs }} \\
\chi_{\text {red }}^{2}\end{array}$ & $\begin{array}{l}F_{x, \text { unab }} \\
L_{x, \text { unab }}\end{array}$ \\
\hline Chandra & $0.7 \pm 0.1$ & $1.4 \pm 0.3 \times 10^{-12}$ & $3.5_{-0.9}^{+1.4} \times 10^{-12}$ & $4.2_{-0.8}^{+1.0}$ & $1.5_{-0.3}^{+0.4} \times 10^{-12}$ & $3.3_{-2.7}^{+24.4} \times 10^{-10}$ \\
\hline 2007-06-13 & $5.4_{-1.4}^{+1.6}$ & 0.7 & $3.4_{-0.9}^{+1.4} \times 10^{34}$ & $10.5_{-2.1}^{+2.5}$ & 0.7 & $3.2_{-2.6}^{+23.9} \times 10^{36}$ \\
\hline Chandra & $0.7 \pm 0.1$ & $1.8_{-0.2}^{+0.3} \times 10^{-13}$ & $4.9_{-1.2}^{+2.2} \times 10^{-13}$ & $4.3_{-0.8}^{+0.9}$ & $2.1_{-0.3}^{+0.4} \times 10^{-13}$ & $5.9_{-4.9}^{+45.1} \times 10^{-11}$ \\
\hline 2009-06-14 & $5.4_{-1.4}^{+1.6}$ & & $4.8_{-1.2}^{+2.2} \times 10^{33}$ & $10.5_{-2.1}^{+2.5}$ & & $5.7_{-4.8}^{+43.8} \times 10^{35}$ \\
\hline Chandra & $0.8_{-0.2}^{+0.3}$ & $1.6_{-0.4}^{+0.6} \times 10^{-13}$ & $3.6_{-0.9}^{+1.6} \times 10^{-13}$ & $3.6_{-1.1}^{+1.2}$ & $2.0_{-0.6}^{+0.9} \times 10^{-13}$ & $1.3_{-1.1}^{+12.6} \times 10^{-11}$ \\
\hline 2009-07-10 & $5.4_{-1.4}^{+1.6}$ & & $3.5_{-0.9}^{+1.6} \times 10^{33}$ & $10.5_{-2.1}^{+2.5}$ & & $1.2_{-1.0}^{+12.2} \times 10^{35}$ \\
\hline$X M M$ & $0.5 \pm 0.1$ & $3.0_{-0.6}^{+0.8} \times 10^{-14}$ & $1.1_{-0.4}^{+0.9} \times 10^{-13}$ & $5.4_{-1.1}^{+1.3}$ & $3.2_{-0.8}^{+0.9} \times 10^{-14}$ & $7.9_{-7.1}^{+133.3} \times 10^{-11}$ \\
\hline $2011-02-22$ & $5.4_{-1.4}^{+1.6}$ & & $1.1_{-0.4}^{+0.9} \times 10^{33}$ & $10.5_{-2.1}^{+2.5}$ & & $7.7_{-6.9}^{+129.4} \times 10^{35}$ \\
\hline
\end{tabular}

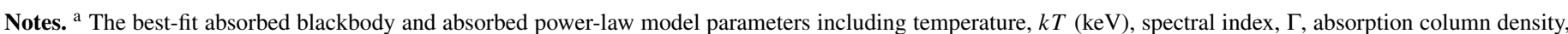

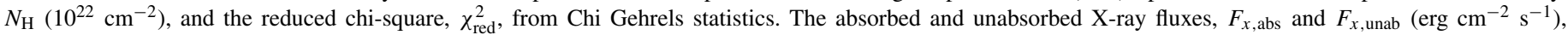

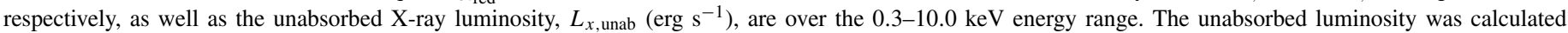
assuming a distance of $9 \mathrm{kpc}$ (Levin et al. 2010). All fit parameter errors are for $90 \%$ confidence.

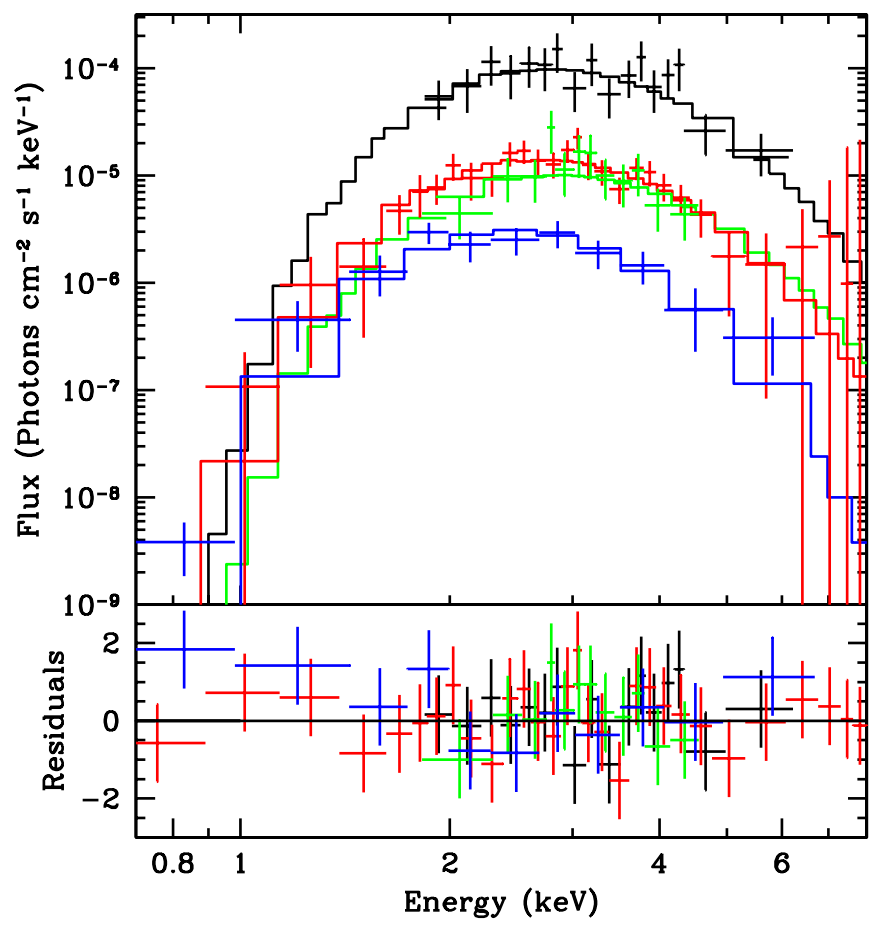

Figure 1. 2007 June (black), 2009 June (red), and 2009 July (green) Chandra spectra and 2011 February (blue) XMM spectrum of PSR J1622-4950. These spectra were fitted simultaneously with an absorbed black body model with $N_{\mathrm{H}}$ locked between epochs but with $k T$ and the normalization values for each spectra allowed to vary individually. The bottom panel shows the residuals of these fits.

(A color version of this figure is available in the online journal.)

calculating the flux and $90 \%$ flux error for different energy bands (e.g., 0.3-2 keV, 2-4 keV, etc). The flux hardness ratios for different energy band combinations (soft/hard), propagating through the $90 \%$ errors, show no evidence of spectral variation between the epochs. While this method is model dependent it also demonstrates that there is no strong evolution to PSR J1622-4950's X-ray spectrum.

Levin et al. (2010) calculated a dispersion measure (DM) distance of $9 \mathrm{kpc}$ to PSR J1622-4950 using the Cordes \&
Lazio (2002) NE2001 Galactic free electron density model. ${ }^{30}$ We used this distance to estimate the unabsorbed $0.3-10.0 \mathrm{keV}$ luminosity at the four epochs from the unabsorbed fluxes calculated from both the blackbody and power-law spectral fits. These values are summarized in Table 3. The X-ray luminosities calculated from the blackbody fit show a monotonic reduction over the four epochs and are consistent with the range of luminosities seen from other magnetars. ${ }^{31}$ However, the luminosities calculated from the power-law fit are on the high end of known magnetar luminosities, with the large errors preventing us from observing any obvious evolution between epochs.

\subsubsection{X-Ray Timing Analysis}

Only the $X M M$ data were used in our X-ray timing investigation as our Chandra/ACIS observations do not have sufficient time resolution to detect PSR J1622-4950's pulse period of $4.3 \mathrm{~s}$. Using the EPIC-PN observations, which have a time resolution of $73.4 \mathrm{~ms}$, we shifted all photons arrival times to the solar system barycenter, and used the XRONOS software for the timing analysis. We used data in the $0.3-4.0 \mathrm{keV}$ energy range where the source was relatively bright, then folded the data using the pulsar ephemeris derived from ongoing radio monitoring: $P=4.32645312$ s at Modified Julian Date (MJD) 55586.5 (Levin et al. 2012). We do not detect a significant signal in the X-ray band with a $3 \sigma$ upper limit on the 0.3-4.0 keV pulsed fraction of 70\% (as derived by Vaughan et al. 1994; Israel \& Stella 1996). The pulsed fraction is defined as $\left(N_{\max }-N_{\min }\right) /\left(N_{\max }+N_{\min }\right)$, with $N_{\max }$ and $N_{\min }$ being the maximum and minimum counts of a putative sinusoidal signal at the frequency of PSR J1622-4950. A similar analysis was also conducted in the $0.3-2.0$ and $2.0-10.0 \mathrm{keV}$ energy bands as the strength and phase of a magnetar's pulsations can be energy dependent (Gotthelf \& Halpern 2009). Each band had $<100$ counts with no pulsations detected, resulting in an unconstraining pulse fraction upper limit of $99 \%$.

\footnotetext{
30 This distance estimate should be treated with caution as proper motion studies have shown that DM-based distance measurements can differ by more than a factor of two to the distance calculated from parallax (Chatterjee et al. 2009; Deller et al. 2009)

31 See http://www.physics.mcgill.ca/ pulsar/magnetar/main.html.
} 

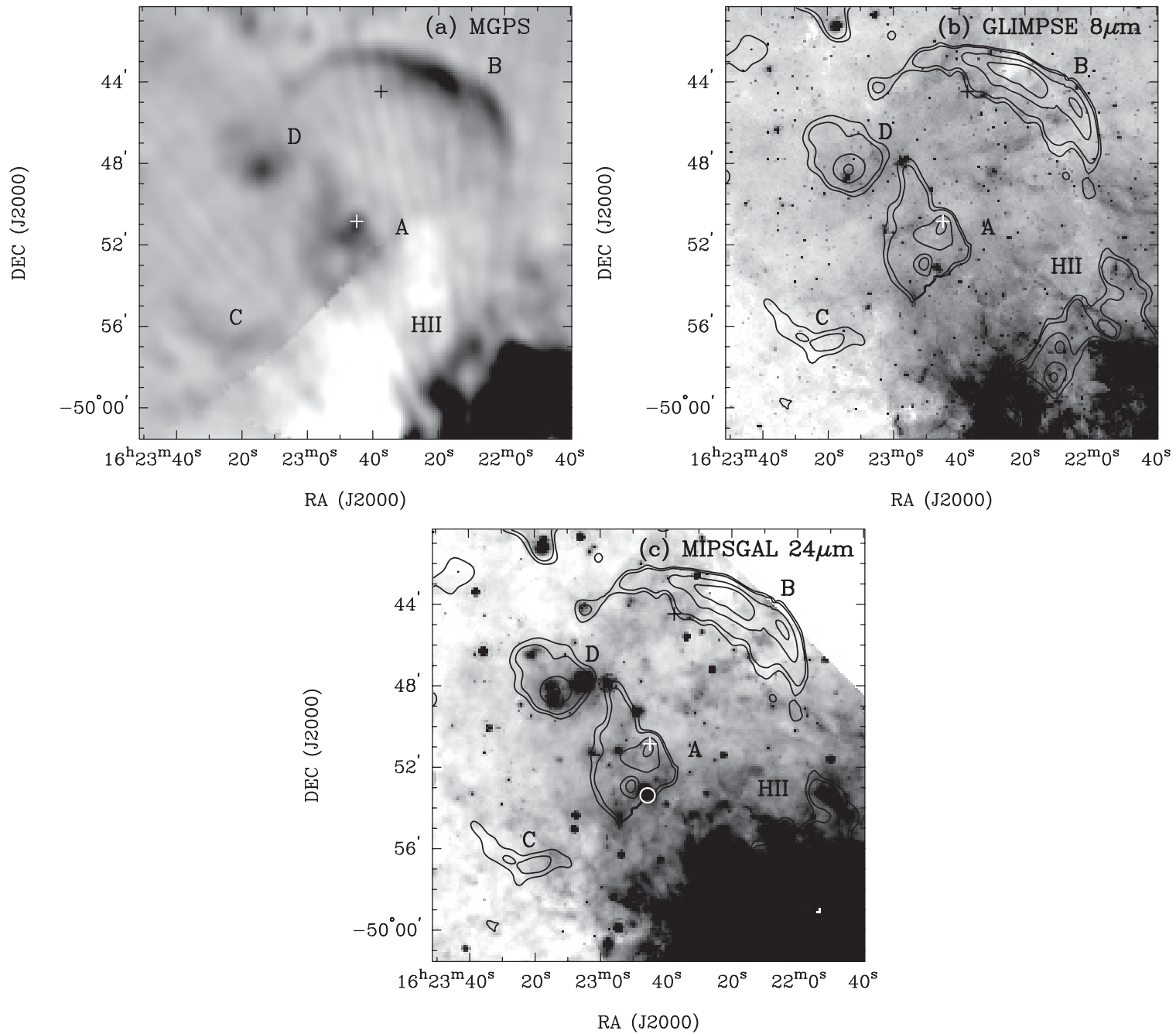

Figure 2. Radio and infrared images of the region surrounding PSR J1622-4950. Each image is centered on the position of PSR J1622-4950, indicated by the white "+" symbol. Nearby radio sources are labeled A, B, C, and D, and the Hil region, G333.6-0.2, is also indicated. The black contours in panels (b) and (c) show the MGPS1 $0.005,0.01,0.03$, and $0.05 \mathrm{mJy}_{\text {beam }}^{-1}$ radio emission levels. The nearby pulsar, PSR J1622-4944, is indicated by a black " + " symbol. (a) The surrounding region as seen in the MGPS1 radio survey at $843 \mathrm{MHz}$ and at a resolution of $43^{\prime \prime}$. (b) The gray scale is the surrounding region as seen in GLIMPSE at $8 \mu \mathrm{m}$ at a resolution of $1^{\prime \prime} .2$. (c) The gray scale as seen by MIPSGAL at $24 \mu \mathrm{m}$ at a resolution of $6^{\prime \prime}$. The white circle indicates the position of IRAS $16190-4946$ with a radius of $23^{\prime \prime}$, equivalent to its major axis position uncertainty.

In order to search for short term variability from PSR J1622-4950 in the three Chandra observations we corrected the photon arrival times to the solar system barycenter. In the case of the 2007 June observation we also filtered out those times when the source was not on the chip to remove any contribution caused by the source dithering on and off the chip. There was no evidence of short term variability beyond $40 \%$ of the mean count rate between $250 \mathrm{~s}$ and $2020 \mathrm{~s}, 5000 \mathrm{~s}$ and $60,870 \mathrm{~s}$, and $2500 \mathrm{~s}$ and 20,130 s in the 2007 June, 2009 June and 2009 July Chandra observations, respectively. ${ }^{32}$

\subsection{Radio Results}

\subsubsection{Archival}

The region surrounding PSR J1622-4950 at radio wavelengths in MGPS1 is shown in Figure 2(a). The position of PSR J1622-4950 is denoted by a white "+" sign. The diffuse radio emission surrounding PSR J1622-4950, encompassed by the contours seen in Figures 2(b) and (c), is denoted as source A.

\footnotetext{
32 The smaller number in each range is the size of a time bin chosen to include a statistically significant number of counts. The upper number is the exposure time.
}

There is a nearby $\mathrm{H}$ II region, G333.6-0.2, located to the southwest of the pulsar (Paladini et al. 2003). The other three diffuse radio sources in the immediate vicinity of the pulsar are denoted B, C, and D. Figures 2(b) and (c) show the corresponding infrared GLIMPSE $8 \mu \mathrm{m}$ and MIPSGAL $24 \mu \mathrm{m}$ views of this area of sky, respectively, overlaid with MGPS 1 as black contours.

The radio source A does not appear to have been cataloged in any surveys of this region. The brightest knot of radio emission in source A is coincident with PSR J1622-4950 and extends out in some directions as far as $4^{\prime}$. This diffuse radio emission also forms a ring morphology below PSR J1622-4950, which can be seen in Figure 2(a). This same morphology was also resolved by Levin et al. (2010) using ATCA. Faint diffuse infrared emission is detected all over the immediate field as seen in Figures 2(b) and (c), but is not concentrated in the vicinity of the bright knot of radio emission in source A. It is therefore more likely that this diffuse infrared emission originates from G333.6-0.2, which is extremely bright in the radio and at 8 and $24 \mu \mathrm{m}$. At the southern edge of source A there is a very bright $24 \mu \mathrm{m}$ source, IRAS 16190-4946 (ellipse major and minor axis uncertainties of $23^{\prime \prime}$ and $3^{\prime \prime}$, respectively; Helou \& Walker 1988 ), indicated by a white $23^{\prime \prime}$ radius circle in Figure 2(c). 
IRAS 16190-4946 lies $\sim 2^{\prime}$ from PSR J1622-4950 so it is unlikely to be associated with source A immediately coincident with the magnetar.

To the northwest of the pulsar there is a radio source in the shape of an arc, labeled source B in Figure 2. Figures 2(b) and (c) show no evidence for diffuse infrared emission matching the structure of source B. If we assume that this arc is part of a circular shell, we measure an equivalent radius of $7^{\prime} \pm 1^{\prime}$ with approximate central coordinates of (J2000) R.A. $=16: 22: 38$ and decl. $=-49: 49: 48$. The inferred center of the arc is offset 0.8-2'.5 from the position of PSR J1622-4950. Another pulsar, PSR J1622-4944, indicated by a black "+" sign in Figure 2, lies $1^{\prime}$ inside the inner edge of the arc. The distance to PSR J1622-4944 is $\sim 7.9 \mathrm{kpc}$ calculated using the DM published by Manchester et al. (2001) assuming the Cordes \& Lazio (2002) model. There is also an infrared dark cloud, SDC G333.900+0.022 (Peretto \& Fuller 2009), coincident with the arc, but it is unlikely to be associated given that radio emission is not commonly associated with such clouds and that its morphology does not match that of source B.

A possible counterarc to source $\mathrm{B}$, labeled source $\mathrm{C}$ in Figure 2, is seen $\sim 8^{\prime}$ to the southeast of PSR J1622-4950, but the lack of symmetry between these arcs renders an association between the two speculative. Radio source D is coincident with the 6.7 GHz maser source G333.93-0.13 (Pestalozzi et al. 2005) and is likely also associated with IRAS 16194-4941. At $24 \mu \mathrm{m}$ other infrared sources are detected within the brighter radio contours of source D, which are very faint or undetected at $8 \mu \mathrm{m}$.

\subsubsection{ATCA Results}

Initial analysis of the 2008 November and 2008 December ATCA data demonstrated that we did not have enough sensitivity to see the diffuse emission surrounding PSR J1622-4950 as seen in MGPS, SGPS, and the Levin et al. (2010) ATCA observations (which took place on 2009 December 8 and 2010 February 27). While the Levin et al. (2010) ATCA observations used the same frequencies, array configurations, and integration times as the 2008 ATCA observations presented here, they were using the new ATCA correlator, which has a $2 \mathrm{GHz}$ bandwidth and thus a much higher sensitivity. Regardless of the lack of diffuse emission, our ATCA observations did detect a radio point source at the magnetar's Chandra position. We analyzed the 2008 November and 2008 December observations separately, using just the antenna 6 baselines (i.e., baselines between 5 and $6 \mathrm{~km})$. This significantly reduced sidelobe contamination from G333.6-0.2.

The resulting time and phase averaged flux densities and polarizations of PSR J1622-4950, measured during the 2008 November and 2008 December ATCA observations, are listed in Table 2 . While the $9 \mathrm{GHz}$ flux density remained fairly steady between the two observations, it increased by $22 \%$ at $5 \mathrm{GHz}$. The ATCA observations of PSR J1622-4950 taken by Levin et al. (2010) (see Table 2) indicate a decrease in radio flux of $\sim 68 \%$ and $\sim 55 \%$, at 5 and $9 \mathrm{GHz}$ respectively, approximately one year after our 2008 December ATCA observation.

The flux changes we observed between our 2008 November and 2008 December ATCA observations demonstrated a steepening in the radio spectral index of PSR J1622-4950 over two weeks, from $\alpha=-0.13 \pm 0.04$ on 2008 November 22 to $\alpha=-0.44 \pm 0.04$ on 2008 December 5, where $S_{v} \propto v^{\alpha}$. These values contrast to the positive time-averaged spectral index derived by Levin et al. (2010), calculated using a combination of observations with different telescopes in the frequency range 1.4-9 GHz and over various epochs between 1998 February and 2010 January.

The polarization of PSR J1622-4950 also changed significantly between our two ATCA epochs. During the 2008 November ATCA observation the linearly polarized fraction was $\sim 80 \%$ and circular polarization $\lesssim 8 \%$ at both frequencies. Two weeks later the linearly polarized fraction had dropped to $<20 \%$ but the source had become $\sim 15 \%$ circularly polarized. (This circular polarization was negatively handed using the pulsar astronomy sign convention.) It should be noted that these polarization fractions quoted here are lower limits on the true peak polarization of PSR J1622-4950 given that the ATCA observations of the pulse signal are time and phased averaged.

\section{DISCUSSION}

\subsection{Variability}

The spin-down luminosity of PSR J1622-4950 calculated by Levin et al. (2010) is $\dot{E}=8.5 \times 10^{33} \mathrm{erg} \mathrm{s}^{-1}$. Using the unabsorbed blackbody luminosities listed in Table 3 we find that $L_{x} \sim 3.5 \dot{E}, L_{x} \sim 0.6 \dot{E}, L_{x} \sim 0.5 \dot{E}$, and $L_{x} \sim 0.1 \dot{E}$ for the 2007 June, 2009 June, 2009 July Chandra, and 2011 February $X M M$ observations, respectively, in the $0.3-10.0 \mathrm{keV}$ energy range. All these ratios are significantly higher than what we expect from young pulsars but are similar to what we see from other magnetars. ${ }^{33}$ One of the defining magnetar characteristics is $L_{x}>\dot{E}$ (Mereghetti 2008) but this relationship can also be the case for cooling neutron stars (for example see van Kerkwijk \& Kaplan 2008; Halpern \& Gotthelf 2011). However, the X-ray variability and relatively high X-ray luminosity that we observe from PSR J1622-4950 are not consistent with cooling. We therefore confirm the Levin et al. (2010) identification of PSR J1622-4950 as a magnetar.

The simultaneous blackbody and power-law spectral fits to our four X-ray spectra of PSR J1622-4950 show that over 3.7 years the X-ray flux decreased by a factor of 47 . Figure 3 is a light curve showing the blackbody absorbed X-ray flux values in blue where the Chandra observations are denoted by open circles and the $X M M$ observation is a filled circle. Figure 3 clearly illustrates the smooth decay of the X-ray emission from an outburst that may have occurred before or during the 2007 June Chandra observation. We therefore suggest that PSR J1622-4950 is a transient magnetar, having demonstrated similar variations in X-ray flux to the two transient magnetars XTE J1810-197 and 1E 1547.0-5408 (Gotthelf \& Halpern 2005, 2007; Bernardini et al. 2009; Halpern et al. 2008).

The decays of outbursts from transient magnetars have been modeled as exponentials, power laws, and multiple power laws (for example, see Figure 3 of Rea \& Esposito 2011). We fitted both an exponential and power law to the absorbed blackbody $\mathrm{X}$-ray flux values from the Chandra and XMM observations. (We determined from these X-ray observations that the crosscalibration between the instruments was no larger than $12 \%$, which is within the $90 \%$ confidence flux errors.) The decay of the X-ray light curve is best described by an exponential with a characteristic decay time of $\tau \sim 360 \pm 11$ days. This is similar to the X-ray decay of XTE J1810-197 (Bernardini et al. 2009) but much slower than the decay of the multiple bursts produced by $1 \mathrm{E}$ 1547.0-5408 (for example see Israel et al. 2010).

\footnotetext{
33 Typical $\dot{E}$ and $L_{x}$ values for most magnetars are summarized at http://www.physics.mcgill.ca/ pulsar/magnetar/main.html.
} 


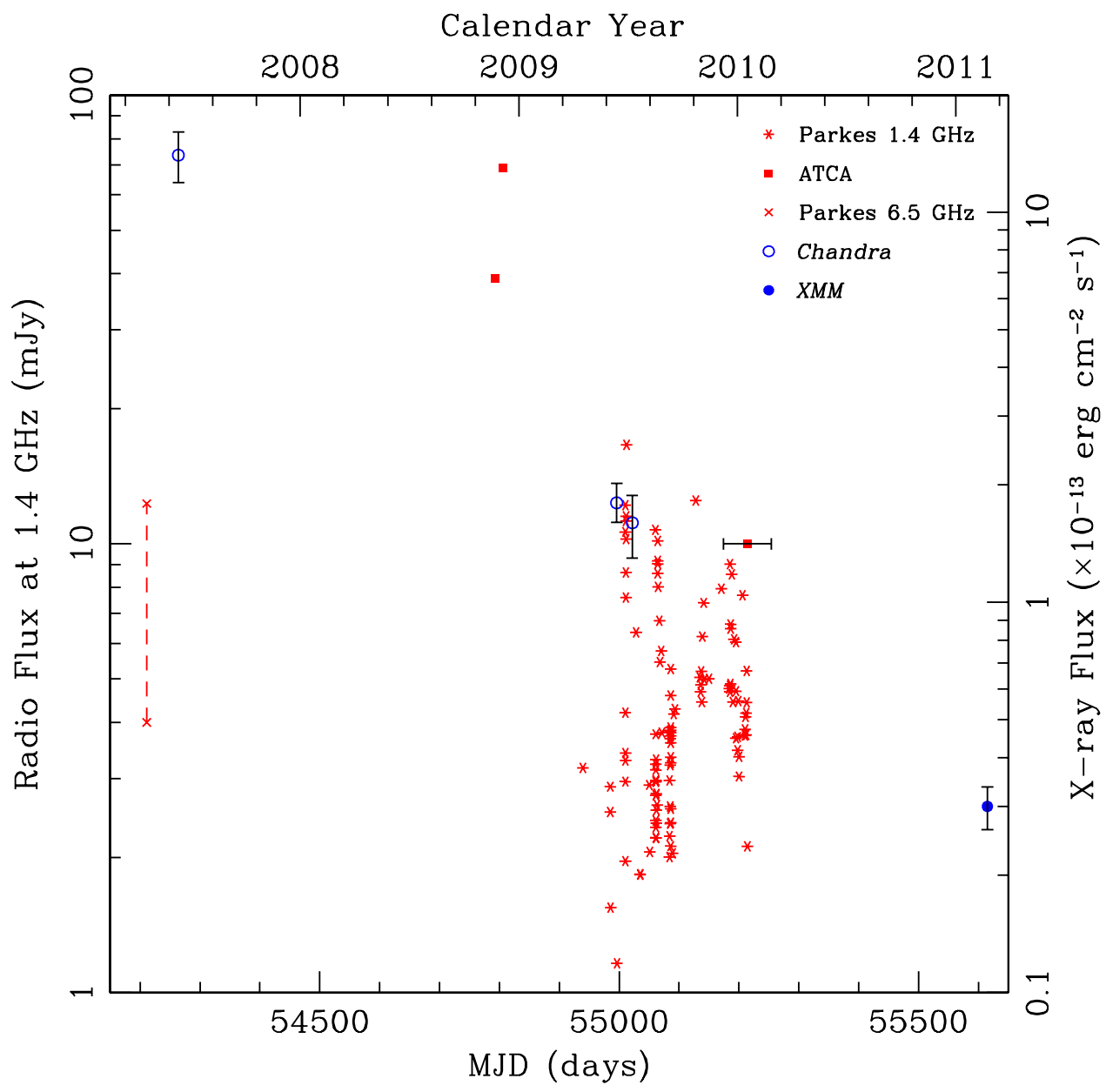

Figure 3. Radio and X-ray light curves of PSR J1622-4950 over 1500 days. The left vertical axis shows the radio flux at $1.4 \mathrm{GHz}$ in units of mJy and the right vertical axis shows the absorbed X-ray flux in units of $10^{-13} \mathrm{erg} \mathrm{cm}^{-2} \mathrm{~s}^{-1}$ in the $0.3-10.0 \mathrm{keV}$ energy range. The radio flux points are in red where the Parkes $1.4 \mathrm{GHz}$ data are denoted by asterisks and the Parkes $6.5 \mathrm{GHz}$ Multibeam pulsar survey measurement is a range of possible values at $1.4 \mathrm{GHz}$, calculated from a range of possible radio spectral indices, denoted by two crosses connected by a dashed line. (These light-curve points were originally depicted in Figure 1 of Levin et al. 2010.) The red squares show the ATCA detections of PSR J1622-4950 when extrapolated to $1.4 \mathrm{GHz}$. The ATCA detection with the horizontal error bar is the average flux value from two ATCA observations, taken in 2009 December and 2010 February, published by Levin et al. (2010). The ATCA flux errors are the size of the data point. The X-ray flux points are in blue where the Chandra observations are denoted by open circles and the $X M M$ observation by a filled circle. The error bars are $1 \sigma$. The upper limit on the X-ray flux of PSR J1622-4950 detected by ASCA in 1999 April (MJD 51291) was $F_{x} \leqslant 3 \times 10^{-13} \mathrm{erg} \mathrm{cm}^{-2} \mathrm{~s}^{-1}$.

(A color version of this figure is available in the online journal.)

In Section 3.1.2, we demonstrated that there is no evidence of strong evolution in PSR J1622-4950's X-ray spectrum over the 3.7 years of X-ray observations. A consistent temperature between epochs was also observed from XTE J1810-197, where the blackbody temperature remained unchanged for over three years after the initial outburst (Bernardini et al. 2009). Further $\mathrm{X}$-ray observations with higher count statistics are required to determine if the X-ray spectrum of PSR J1622-4950 is evolving with time.

If the fading X-ray emission that we have observed from PSR J1622-4950 is thermal, then it is likely emitted by a hot spot on the surface of the magnetar that remains constant in temperature but decreases in size over time. This behavior is potentially explained by the magnetar coronal model (Beloborodov \& Thompson 2007). Specifically Gotthelf \& Halpern (2005) suggest that the shrinking of a hot spot after a burst could be due to the decay of currents or rearrangement of the magnetic field lines altering the heat being channeled to the surface. The changes in X-ray flux on the order of weeks to years may be the magnetar's crust plastically responding to the unwinding of these fields, which in turn deposits energy into the magne- tosphere resulting in transient behavior (Muno et al. 2007 and references therein).

The radio spectral index of PSR J1622-4950 (listed in Table 2) appears to be variable and far flatter than the steep, $\alpha \approx-1.6$, stable average spectral index we expect from ordinary young radio pulsars (Lorimer et al. 1995). It is, however, very similar in behavior to the other radio magnetars, XTE J1810-197 and 1E 1547.0-5408 (Camilo et al. 2007c; Lazaridis et al. 2008; Camilo et al. 2008). The time-variable radio spectral indices of magnetars are not well understood but Thompson (2008a, 2008b) suggests that this phenomenon could be the result of current-driven instabilities in the closed magnetosphere of the magnetar. Serylak et al. (2009 and references therein) also speculate that the observed flat radio spectra of magnetars could be the result of the open magnetic field lines having a high plasma density.

As mentioned in Section 2.2, our PANIC observations obtained a counterpart lower limit of $K_{s} \geqslant 20.7$ for PSR J1622-4950. Currently only seven magnetars have been detected in the $K_{s}$ band (Rea \& Esposito 2011). By correcting for the difference in extinction and relative distances, a 
$K_{s}$-band counterpart similar to that seen from the majority of these infrared magnetars, at the position of PSR J1622-4950, would be extremely faint, having a magnitude $>24.7 .{ }^{34}$ While the variable infrared behavior is not consistent between magnetars the ratio between their X-ray and infrared flux appears to remain fairly consistent where $F_{v} v \simeq 1 \times 10^{4} F_{x}$ (Durant \& van Kerkwijk 2005), predicting a $K$-band magnitude of 24.5 for PSR J1622-4950 based on the 2007 June Chandra observation. A much deeper infrared observation is therefore required to detect the counterpart to PSR J1622-4950 during a future period of X-ray bursting activity.

\subsection{Polarization}

In Section 3.2.2, we showed that the linearly and circularly polarized fractions of the radio emission from PSR J1622-4950 both changed significantly between 2008 November and 2008 December. In contrast, phase resolved radio observations of the two radio magnetars XTE J1810-197 and 1E 1547.0-5408 show that their linear polarization is consistently high and that neither their linear or circular polarization exhibit dramatic changes in intensity over time (Kramer et al. 2007; Camilo et al. 2007d, 2008).

The significant linear and circular polarization variability we observed from PSR J1622-4950 can be explained by changes in the geometry of the magnetosphere of the magnetar causing the overall pulse profile to vary (Camilo et al. 2007a). The 2008 November and 2008 December ATCA observations were phase and time averaged, amplifying the observed polarization variability over the intrinsic behavior. Results from Levin et al. (2010) show that, unlike most normal radio pulsars, the radio pulse profile of PSR J1622-4950 is variable between consecutive epochs, similar to the radio pulse profiles of XTE J1810-197 and 1E 1547.0-5408 (Camilo et al. 2007a; Kramer et al. 2007; Camilo et al. 2008). In Figure 1 of Levin et al. (2010), we see that in three consecutive epochs, the pulse profile shape changed from a double-peaked profile to one in which only the leading peak was detected. Figure 4 of Levin et al. (2010) shows that over the entire pulse profile at 1.4 and $3.1 \mathrm{GHz}$, when both peaks were detected, the polarized position angle (PPA) swung through $180^{\circ}$. If an ATCA observation of PSR J1622-4950 takes place when the entire double-peaked pulse is switched on, and is therefore experiencing a very large PPA swing, phase averaging of the pulse would produce a low linear polarization measurement, such as what we observed in 2008 December. However, if only part of the pulse is switched on, such as in the case of the last pulse profile shown in Figure 1 of Levin et al. (2010), then a phased-averaged observation does not experience the full PPA swing. The phase averaging would then result in less depolarization such as in the case of our 2008 November ATCA observation.

The difference in circular polarization between the two ATCA epochs can be explained by the same changes in the pulse profile. In 2008 December, when we assume that the entire pulse profile is switched on, we see a significant fraction of circular polarization that was not observed in 2008 November. It is possible that the part of the pulse profile that was switched off during the 2008 November observation was the circularly polarized component, resulting in a lack of detectable circularly polarized emission during that epoch. This

\footnotetext{
34 For details on the $K$-band observations and distance estimates of each magnetar see Rea \& Esposito (2011)

http://www.physics.mcgill.ca/ pulsar/magnetar/main.html and references therein.
}

component then switched back on again in 2008 December, allowing us to detect circular polarization. Observations by Levin et al. (2010) have demonstrated that changes in the pulse profile of PSR J1622-4950 happen on the timescale of days and could therefore have occurred in the 14 days between the 2008 November and 2008 December ATCA observations, resulting in the observed polarization variability.

\subsection{Correlation between Time Variability in Radio and X-Rays}

In order to determine if there is any correlation between the X-ray and radio emission from PSR J1622-4950, we have compared our X-ray and ATCA results with the Parkes $1.4 \mathrm{GHz}$ light curve reported by Levin et al. (2010). Figure 3 shows the light curve of PSR J1622-4950 where the radio $1.4 \mathrm{GHz}$ flux values are in red (flux scale on the left axis) and X-ray flux values are in blue (flux scale on the right axis). The radio flux points include the Parkes measurements from Levin et al. (2010) and our ATCA detections extrapolated to $1.4 \mathrm{GHz}$. This extrapolation assumes that the radio spectral index of PSR J1622-4950, calculated from these ATCA observations (see Table 2), describes the radio spectrum down to low frequencies, just as in the case of XTE J1810-197 (Lazaridis et al. 2008). This results in estimated $1.4 \mathrm{GHz}$ fluxes of $\sim 39 \mathrm{mJy}$ during the 2008 November observation and $\sim 69$ mJy during the 2008 December observation, which are far brighter than any other radio detection of PSR J1622-4950 at $1.4 \mathrm{GHz}$. If our values represent the fluxes of PSR J1622-4950 at $1.4 \mathrm{GHz}$ on these two dates then this magnetar was undergoing a radio flaring event during our ATCA observations and was therefore in a high radio state. All other radio measurements, including those at earlier epochs than covered by the time range of Figure 3 (see Figure 1 of Levin et al. 2010), have revealed considerably lower flux values or only upper limits.

The 2007 June (MJD 54264) Chandra observation detected PSR J1622-4950 in a reasonably high X-ray flux state when the magnetar was likely recovering from a recent X-ray outburst. The 2008 November and 2008 December ATCA observations then indicate a high radio flux from PSR J1622-4950 1.45 years after this Chandra observation (assuming that the spectral index from the ATCA observations can be extrapolated to $1.4 \mathrm{GHz}$ ). Studies of XTE J1810-197 and 1E J1547.0-5408 show that their radio emission was trigged by an X-ray outburst (Halpern et al. 2005; Camilo et al. 2007b) and, in the case of XTE J1810-197, this radio emission peaked in intensity $\sim 3$ years after its X-ray outburst (Camilo et al. 2006). Therefore, it is possible that the high radio state observed for PSR J1622-4950 with ATCA in 2008 was triggered by the X-ray outburst that occurred around the time of the 2007 June Chandra observation.

The 2009 June and 2009 July Chandra observations show that the X-ray flux declined by a factor of eight following the 2007 June Chandra observation. The 2009 June and 2009 July Chandra observations occurred during the period between MJD 54900 and MJD 55250 for which the Parkes observations showed the $1.4 \mathrm{GHz}$ flux to be extremely variable (see Figure 3 ). The XMM observation shows that by 2011 February the X-ray flux had declined by an additional factor of six. Further $\mathrm{X}$-ray and radio observations are required to determine whether PSR J1622-4950 has subsequently returned to a quiescent state.

An X-ray flux upper limit of $F_{x} \leqslant 3 \times 10^{-13} \mathrm{erg} \mathrm{cm}^{-2} \mathrm{~s}^{-1}$ (0.3-10.0 keV) was also obtained for PSR J1622-4950 in the 1999 April (MJD 51291) AGPS observation (see Section 3.1.1). Figure 1 of Levin et al. (2010) indicates that Parkes did not 
detect PSR J1622-4950 at $1.4 \mathrm{GHz}$ before, during, or shortly after this $A S C A$ observation. It was not until 50 days after the ASCA observation that Parkes detected radio emission. Unfortunately, without further X-ray flux history, it is not possible to determine whether the X-ray detection is real and/or related to the subsequent radio detection.

\section{4. $\gamma$-Ray Counterpart}

PSR J1622-4950 falls within the 95\% error circle of the Fermi Large Area Telescope (LAT) source 0FGL J1622.4-4945 (Abdo et al. 2009). 0FGL J1622.4-4945 was one of the FermiLAT bright $\gamma$-ray sources (Abdo et al. 2009), detected in the first three months of observations, with a statistical significance $\geqslant 10 \sigma$. However, OFGL J1622.4-4945 is one of 10 Fermi-LAT Bright Source List sources that were not detected in the FermiLAT First Source Catalog (1FGL), a more recent catalog in which each source detection is based on the average flux over an 11 month period with a statistical significance $\geqslant 4 \sigma$ (Abdo et al. 2010a). This is not unexpected due to its location in the Galactic ridge, since Fermi sources in this region are far more difficult to detect and characterize in the 1FGL catalog analysis (Abdo et al. 2010a).

PSR J1622-4950 lies $\sim 5.8$ from the centroid of OFGL J1622.4-4945 so it is worth investigating if there is an association between these two objects. Fermi $\gamma$-ray pulsars have been found to have spin-down luminosities between $\dot{E}=3 \times 10^{33}$ and $5 \times 10^{38} \mathrm{erg} \mathrm{s}^{-1}$ (Abdo et al. 2010c). While the spin-down luminosity of PSR J1622-4950 is encompassed in this range, this magnetar is far more distant than the $\gamma$-ray pulsars listed in Abdo et al. (2010c), the majority of which lie within $3 \mathrm{kpc}$ of Earth. The Fermi $\gamma$-ray pulsars have an $\dot{E} / d^{2}$ between $3 \times 10^{33}$ and $1 \times 10^{38} \mathrm{erg} \mathrm{s}^{-1} \mathrm{kpc}^{-2}$ (Abdo et al. 2010c). The value of $\dot{E} / d^{2}$ for PSR J1622-4950 is $1 \times 10^{32} \mathrm{erg} \mathrm{s}^{-1} \mathrm{kpc}^{-2}$, an order of magnitude smaller than the minimum value seen from the Fermi pulsars. An association between OFGL J1622.4-4945 and PSR J1622-4950 is therefore unlikely, particularly as an investigation of much closer magnetars did not yield a $\gamma$-ray detection with Fermi-LAT (Abdo et al. 2010b).

\subsection{Supernova Remnant Association}

Magnetars are young neutron stars. However, while the number of identified magnetars is increasing, few have been found to have a convincing association with a SNR: 1E $2259+586$ in CTB 109 (Fahlman \& Gregory 1981), 1E 1841-045 in Kes 73 (Vasisht \& Gotthelf 1997), 1E 1547.0-5408 in SNR G327.24-0.13 (Gelfand \& Gaensler 2007), and the yetto-be-confirmed magnetars AX J1845-0258 in SNR G29.6+0.1 (Gaensler et al. 1999) and CXOU J171405.7-381031 in CTB 37B (Aharonian et al. 2008; Halpern \& Gotthelf 2010). ${ }^{35}$ Such associations are important because SNRs provide independent constraints on the environment and the properties of the associated magnetar.

Source B (and its possibly associated counterarc source C) appears to have similar properties to SNRs as the apparent lack of associated diffuse infrared emission, as mentioned in Section 3.2.1, implies a non-thermal nature (for example, see Brogan et al. 2006). PSR J1622-4950 also resides within 2'.5 of the center of the extrapolated structure. Another pulsar, PSR J1622-4944, mentioned in Section 3.2.1, lies $\sim 1^{\prime}$ from Source B but has a high characteristic age of $10^{6} \mathrm{yr}$ (Manchester

\footnotetext{
35 Another possible association is SGR 0526-66 with SNR N49 in the Large Magellanic Cloud (Cline et al. 1982; Gaensler et al. 2001; Park et al. 2012).
}

et al. 2001). While it is true that a pulsar's characteristic age can be overestimated, it is extremely unusual for a pulsar with such a high characteristic age to be found associated with an SNR. In the absence of any further evidence, PSR J1622-4944 appears to be an ordinary old radio pulsar unrelated to source $B$.

Confirmation of source B as an SNR requires the measurement of a non-thermal radio spectral index or the detection of linear polarization, neither of which is possible to obtain with our current data sets. (SGPS lacks the sensitivity required to disentangle the diffuse sources in this complicated region and the MGPS does not have any polarization information. The frequencies of both these surveys are also too closely spaced to provide a meaningful spectral index estimation.) Instead we need to consider the feasibility of such an identification by exploring a possible connection between source B and PSR J1622-4950 using some of the pulsar/SNR association criteria established by Kaspi (1996). In the following discussion the criteria we explore are whether PSR J1622-4950 and G333.9+0.0 have consistent ages and if the implied transverse velocity of the magnetar away from the assumed explosion site is reasonable. As we do not know the distance to G333.9+0.0 we cannot investigate whether the distances to the magnetar and SNR are consistent. For the purposes of further consideration we assume a common distance of $9 \mathrm{kpc}$ to both sources. The probability of chance alignment between the magnetar and SNR also needs to be considered as demonstrated by Gaensler et al. (2001). We designate source B as G333.9+0.0 based on its approximate centroid and will assume it is an SNR in our discussion below.

The positional coincidence of PSR J1622-4950 with the center of G333.9+0.0 could suggest a possible association. The chance probability of finding an arc in MGPS1 whose emission is non-thermal (based on comparisons with GLIMPSE data), and whose center is within 2.5 of a given position on the sky, is about $5 \%$ based upon inspecting 100 random positions for $315 \leqslant l \leqslant 357$ and $|b| \leqslant 0.4$. This probability is not particularly high or low, and so does not strengthen or argue against an association.

The angular separation between the estimated center of the arc G333.9+0.0 and the position of the PSR J1622-4950 is 0'.8-2'.5. Using PSR J1622-4950's characteristic age of $4 \mathrm{kyr}$ (Levin et al. 2010), this leads to a projected velocity of $500-1500 \mathrm{~km} \mathrm{~s}^{-1}$ for a distance of $9 \mathrm{kpc}$. This is consistent with the overall velocity distribution for pulsars (Arzoumanian et al. 2002), but higher than that observed or inferred for magnetars (Gaensler et al. 2001; Helfand et al. 2007; Kaplan et al. 2009; Deller et al. 2012; Tendulkar et al. 2012).

No X-ray emission is detected from G333.9+0.0 in the XMM observation; the upper limit on the count rate is 0.04 counts $\mathrm{s}^{-1}$ using standard errors and roughly accounting for vignetting (Romer et al. 2001). Assuming a thin-shell morphology and the Sedov-Taylor solution (Sedov 1946a, 1946b; Taylor 1950a, 1950b), we find that the upper limit implies a preshock ambient density lower than $n_{0}=0.05 \mathrm{~cm}^{-3}$ for an explosion energy of $10^{51} \mathrm{erg}$. For an SNR with an $18 \mathrm{pc}$ radius this ambient density predicts a swept up mass of $30 M_{\odot}$, supporting Sedov-Taylor expansion. Here, we have used the standard shock jump conditions for an ideal gas to determine the gas temperature based on the Sedov-Taylor age for the given value of $n_{0}$, and then estimated the X-ray count rate assuming a Raymond-Smith plasma model in XSPEC. We find a Sedov-Taylor upper-limit age of $6 \mathrm{kyr}$, at most a factor of 1.5 greater than PSR J1622-4950's characteristic age of $4 \mathrm{kyr}$, making G333.9+0.0 potentially young and similar in age to convincing magnetar/SNR associations 
(Gaensler et al. 2001). This Sedov-Taylor age leads to an upper limit on shock velocity of $\sim 1200 \mathrm{~km} \mathrm{~s}^{-1}$. The associated proton temperature is $<2 \mathrm{keV}$, indicating an electron temperature of $<0.2 \mathrm{keV}$ using the electron-ion equilibration relation from Ghavamian et al. (2007). While the above upper limit on the density is much lower than the mean interstellar medium value, it is not unreasonable for the low-density cavities formed from stellar winds of the massive stars that lead to core-collapse supernovae. We conclude that the radius of the shell and the absence of X-ray emission are both consistent with young SNRs in a low density medium.

The implied non-thermal nature of source A, combined with the positional coincidence of PSR J1622-4950 within its extent, raises the possibility that source $A$ could be a pulsar wind nebula (PWN) generated by PSR J1622-4950. This would be an interesting result, as there is currently no known radio PWN associated with a magnetar. However, a radial profile analysis, comparing the detection of PSR J1622-4950 to the point-spread function of the $X M M$ image, does not show any evidence of an extended X-ray source. We also searched for extended X-ray emission resulting from a dust-scattering halo, similar to that seen around 1E 1547.0-5408 (Tiengo et al. 2010; Olausen et al. 2011), but none was detected. ATCA observations, with longer integration times, are required to determine the true nature of source A.

The above do not provide direct evidence that G333.9+0.0 is the shell of an SNR associated with PSR J1622-4950, but there is no firm evidence to argue against such an association either. Another possible SNR association includes the ring of diffuse radio emission that forms part of source A, which sits $\sim 2^{\prime}$ south of PSR J1622-4950. Levin et al. (2010) discuss the possibility that this ring could be the parent SNR to PSR J1622-4950 as it appears non-thermal in nature given that it lacks an infrared counterpart. However, they consider a link unlikely given the ring's small size and the high implied magnetar birth velocity.

\section{CONCLUSIONS}

In this paper, we have confirmed the Levin et al. (2010) magnetar identification of PSR J1622-4950 through the detection of significant X-ray flux variability and high X-ray luminosity. The high dynamic range in the X-ray flux, combined with the exponential characteristic decay time of $\tau=360 \pm 11$ days, suggests that PSR J1622-4950 may be a new addition to the transient magnetar class and could possibly be recovering from an X-ray outburst that occurred before or during the 2007 June Chandra observation. This X-ray flux variability, along with the variable radio flux and spectral index, makes PSR J1622-4950 similar to the two other known radio magnetars, XTE J1810-197 and 1E 1547.0-5408. Observations with the ATCA show that PSR J1622-4950 may have undergone a radio flaring event approximately one and a half years after the 2007 June Chandra observation, which could have been triggered by the X-ray outburst that occurred around this time in 2007 . The proximity of PSR J1622-4950 to the SNR candidate G333.9+0.0, the implied transverse velocities for PSR J1622-4950, and the apparent young age of SNR G333.9+0.0, all support the possibility of a new magnetar/SNR association.

ASCA observations in 1999 indicate that PSR J1622-4950 may be responsible for some of the X-ray flux detected from AX J162246-4956 in the AGPS (Sugizaki et al. 2001). In the AGPS the magnetars XTE J1810-197, 1E 1547.0-5408, 1E 1841-045 (Vasisht \& Gotthelf 1997), and SGR 1806-20 (Laros et al. 1987; Ulmer et al. 1993) were all detected as X-ray sources.
Through our work in ChIcAGO we anticipate the discovery of other magnetars, which will allow us to further define the properties of this unusual population of neutron stars.

We thank the referee for their careful reading of the manuscript and constructive suggestions. G.E.A acknowledges the support of an Australian Postgraduate Award. B.M.G. acknowledges the support of an Australian Laureate Fellowship. P.O.S. acknowledges partial support from NASA Contract NAS8-03060. N.R. is supported by a Ramon y Cajal Research Fellowship to CSIC, and grants AYA2009-07391 and SGR2009-811, as well as the Formosa Program TW2010005. J.J.D was supported by NASA contract NAS8-39073 to the Chandra X-ray Center (CXC). P.E. acknowledges financial support from the Autonomous Region of Sardinia through a research grant under the program PO Sardegna FSE 2007-2013, L.R. 7/2007. D.T.H.S. acknowledges an STFC Advanced Fellowship. Support for this work was also provided by NASA through Chandra Award Number GO9-0155X issued by the $\mathrm{CXC}$, which is operated by the Smithsonian Astrophysical Observatory for and on behalf of NASA. This research makes use of data obtained with the Chandra X-ray Observatory, and software provided by the CXC in the application packages CIAO. This work is based on observations obtained with XMM-Newton, an ESA science mission with instruments and contributions directly funded by ESA Member States and NASA. MOST is operated with the support of the Australian Research Council and the Science Foundation for Physics within the University of Sydney. ATCA and Parkes, part of the Australia Telescope, are funded by the Commonwealth of Australia for operation as a National Facility managed by CSIRO. Observing time on the $6.5 \mathrm{~m}$ Baade Magellan Telescope, located at Las Campanas Observatory, was allocated through the Harvard-Smithsonian Center for Astrophysics. 2MASS is a joint project of the University of Massachusetts and the IPAC/Caltech, funded by the NASA and NFS. GLIMPSE survey data are part of the Spitzer Legacy Program. The Spitzer Space Telescope is operated by the JPL/Caltech under a contract with NASA. This research has made use of NASA's Astrophysics Data System.

Facilities: ASCA, ATCA, CXO (ACIS), Magellan:Baade (PANIC), Molonglo Observatory, Parkes, XMM (EPIC)

\section{REFERENCES}

Abdo, A. A., Ackermann, M., Ajello, M., et al. 2009, ApJS, 183, 46 Abdo, A. A., Ackermann, M., Ajello, M., et al. 2010a, ApJS, 188, 405 Abdo, A. A., Ackermann, M., Ajello, M., et al. 2010b, ApJ, 725, L73 Abdo, A. A., Ackermann, M., Ajello, M., et al. 2010c, ApJS, 187, 460 Aharonian, F., Akhperjanian, A. G., Barres de Almeida, U., et al. 2008, A\&A, 486, 829

Anderson, G. E., Gaensler, B. M., Kaplan, David L., et al. 2011, ApJ, 727, 105 Arabadjis, J. S., \& Bregman, J. N. 1999, ApJ, 510, 806

Arzoumanian, Z., Chernoff, D. F., \& Cordes, J. M. 2002, ApJ, 568, 289

Balucinska-Church, M., \& McCammon, D. 1992, ApJ, 400, 699

Beloborodov, A. M., \& Thompson, C. 2007, ApJ, 657, 967

Benjamin, R. A., Churchwell, E., Babler, B. L., et al. 2003, PASP, 115, 953

Bernardini, F., Israel, G. L., Dall'Osso, S., et al. 2009, A\&A, 498, 195

Bernardini, F., Israel, G. L., Stella, L., et al. 2011, A\&A, 529, A19

Bertin, E., \& Arnouts, S. 1996, A\&AS, 117, 393

Brogan, C. L., Gelfand, J. D., Gaensler, B. M., Kassim, N. E., \& Lazio, T. J. W. 2006, ApJ, 639, L25

Camilo, F., Cognard, I., Ransom, S. M., et al. 2007a, ApJ, 663, 497

Camilo, F., Ransom, S. M., Halpern, J. P., \& Reynolds, J. 2007b, ApJ, 666, L93

Camilo, F., Ransom, S. M., Halpern, J. P., et al. 2006, Nature, 442, 892

Camilo, F., Ransom, S. M., Peñalver, J., et al. 2007c, ApJ, 669, 561

Camilo, F., Reynolds, J., Johnston, S., Halpern, J. P., \& Ransom, S. M. 2008, ApJ, 679, 681

Camilo, F., Reynolds, J., Johnston, S., et al. 2007d, ApJ, 659, L37 
Carey, S. J., Noriega-Crespo, A., Mizuno, D. R., et al. 2009, PASP, 121, 76 Chatterjee, S., Brisken, W. F., Vlemmings, W. H. T., et al. 2009, ApJ, 698, 250 Cline, T. L., Desai, U. D., Teegarden, B. J., et al. 1982, ApJ, 255, L45 Cordes, J. M., \& Lazio, T. J. W. 2002, arXiv:astro-ph/0207156

Deller, A. T., Camilo, F., Reynolds, J. E., \& Halpern, J. P. 2012, ApJ, 748, L1

Deller, A. T., Tingay, S. J., Bailes, M., \& Reynolds, J. E. 2009, ApJ, 701, 1243

Dickey, J. M., \& Lockman, F. J. 1990, ARA\&A, 28, 215

Dorman, B., \& Arnaud, K. A. 2001, in ASP Conf. Proc. 238, Astronomical Data Analysis Software and Systems X, ed. F. R. Harnden, F. A. Primini, Jr., \& H. E. Payne (San Francisco, CA: ASP), 415

Durant, M., \& van Kerkwijk, M. H. 2005, ApJ, 627, 376

Enoto, T., Nakagawa, Y. E., Rea, N., et al. 2009, ApJ, 693, L122

Evans, I. N., Primini, F. A., Glotfelty, K. J., et al. 2010, ApJS, 189, 37

Fahlman, G. G., \& Gregory, P. C. 1981, Nature, 293, 202

Gaensler, B. M., Gotthelf, E. V., \& Vasisht, G. 1999, ApJ, 526, L37

Gaensler, B. M., Slane, P. O., Gotthelf, E. V., \& Vasisht, G. 2001, ApJ, 559, 963 Garmire, G. P., Bautz, M. W., Ford, P. G., Nousek, J. A., \& Ricker, G. R., Jr. 2003, Proc. SPIE, 4851, 28

Gelfand, J. D., \& Gaensler, B. M. 2007, ApJ, 667, 1111

Ghavamian, P., Laming, J. M., \& Rakowski, C. E. 2007, ApJ, 654, L69

Gotthelf, E. V., \& Halpern, J. P. 2005, ApJ, 632, 1075

Gotthelf, E. V., \& Halpern, J. P. 2007, Ap\&SS, 308, 79

Gotthelf, E. V., \& Halpern, J. P. 2009, ApJ, 695, L35

Green, A. J., Cram, L. E., Large, M. I., \& Ye, T. 1999, ApJS, 122, 207

Halpern, J. P., \& Gotthelf, E. V. 2010, ApJ, 710, 941

Halpern, J. P., \& Gotthelf, E. V. 2011, ApJ, 733, L28

Halpern, J. P., Gotthelf, E. V., Becker, R. H., Helfand, D. J., \& White, R. L. 2005, ApJ, 632, L29

Halpern, J. P., Gotthelf, E. V., Reynolds, J., Ransom, S. M., \& Camilo, F. 2008, ApJ, 676, 1178

Haverkorn, M., Gaensler, B. M., McClure-Griffiths, N. M., Dickey, J. M., \& Green, A. J. 2006, ApJS, 167, 230

Helfand, D. J., Chatterjee, S., Brisken, W. F., et al. 2007, ApJ, 662, 1198

Helou, G., \& Walker, D. W. (ed.) 1988, Infrared Astronomical Satellite (IRAS) Catalogs and Atlases, Vol. 7: The Small Scale Structure Catalog (Washington, DC: NASA)

Hong, J., van den Berg, M., Schlegel, E. M., et al. 2005, ApJ, 635, 907

Israel, G. L., Esposito, P., Rea, N., et al. 2010, MNRAS, 408, 1387

Israel, G. L., \& Stella, L. 1996, ApJ, 468, 369

Kalberla, P. M. W., Burton, W. B., Hartmann, D., et al. 2005, A\&A, 440, 775

Kaplan, D. L., Chatterjee, S., Hales, C. A., Gaensler, B. M., \& Slane, P. O. 2009, AJ, 137, 354

Kaspi, V. M. 1996, in ASP Conf. Ser. 105, IAU Colloq. 160: Pulsars: Problems and Progress, ed. S. Johnston, M. A. Walker, \& M. Bailes (San Francisco, CA: ASP), 375

Keith, M. J., Jameson, A., van Straten, W., et al. 2010, MNRAS, 409, 619

Kramer, M., Stappers, B. W., Jessner, A., Lyne, A. G., \& Jordan, C. A. 2007, MNRAS, 377, 107
Laros, J. G., Fenimore, E. E., Klebesadel, R. W., et al. 1987, ApJ, 320, L111

Lazaridis, K., Jessner, A., Kramer, M., et al. 2008, MNRAS, 390, 839

Levin, L., Bailes, M., Bates, S., et al. 2010, ApJ, 721, L33

Levin, L., Bailes, M., Bates, S., et al. 2012, MNRAS

Lodders, K. 2003, ApJ, 591, 1220

Lorimer, D. R., Yates, J. A., Lyne, A. G., \& Gould, D. M. 1995, MNRAS, 273, 411

Manchester, R. N., Lyne, A. G., Camilo, F., et al. 2001, MNRAS, 328, 17

Martini, P., Persson, S. E., Murphy, D. C., et al. 2004, Proc. SPIE, 5492, 1653

Mereghetti, S. 2008, A\&AR, 15, 225

Monet, D. G., Levine, S. E., Canzian, B., et al. 2003, AJ, 125, 984

Muno, M. P., Gaensler, B. M., Clark, J. S., et al. 2007, MNRAS, 378, L44

Murphy, T., Mauch, T., Green, A., et al. 2007, MNRAS, 382, 382

Olausen, S. A., Kaspi, V. M., Ng, C.-Y., et al. 2011, ApJ, 742, 4

Osip, D. J., Floyd, D., \& Covarrubias, R. 2008, Proc. SPIE, 7014, 70140A

Paladini, R., Burigana, C., Davies, R. D., et al. 2003, A\&A, 397, 213

Park, S., Hughes, J. P., Slane, P. O., et al. 2012, ApJ, 748, 117

Peretto, N., \& Fuller, G. A. 2009, A\&A, 505, 405

Pestalozzi, M. R., Minier, V., \& Booth, R. S. 2005, A\&A, 432, 737

Rea, N., \& Esposito, P. 2011, in High-Energy Emission from Pulsars and Their Systems, ed. D. F. Torres \& N. Rea (Berlin: Springer), 247

Romer, A. K., Viana, P. T. P., Liddle, A. R., \& Mann, R. G. 2001, ApJ, 547, 594

Scholz, P., \& Kaspi, V. M. 2011, ApJ, 739, 94

Sedov, L. I. 1946a, Dokl. Akad. Nauk SSSR, 42, 17

Sedov, L. I. 1946b, Prikl. Mat. Mekh., 10, 241 (No. 2)

Serylak, M., Stappers, B. W., Weltevrede, P., et al. 2009, MNRAS, 394, 295

Skrutskie, M. F., Cutri, R. M., Stiening, R., et al. 2006, AJ, 131, 1163

Strüder, L., Briel, U., Dennerl, K., et al. 2001, A\&A, 365, L18

Sugizaki, M., Mitsuda, K., Kaneda, H., et al. 2001, ApJS, 134, 77

Tam, C. R., Kaspi, V. M., Gaensler, B. M., \& Gotthelf, E. V. 2006, ApJ, 652 , 548

Taylor, G. 1950a, Proc. R. Soc. A, 201, 159

Taylor, G. 1950b, Proc. R. Soc. A, 201, 175

Tendulkar, S. P., Cameron, P. B., \& Kulkarni, S. R. 2012, in American Astronomical Society Meeting Abstracts, Vol. 219, 237.03

Thompson, C. 2008a, ApJ, 688, 1258

Thompson, C. 2008b, ApJ, 688, 499

Tiengo, A., Vianello, G., Esposito, P., et al. 2010, ApJ, 710, 227

Tody, D. 1986, Proc. SPIE, 627, 733

Tody, D. 1993, in ASP Conf. Ser. 52, Astronomical Data Analysis Software and Systems II, ed. R. J. Hanisch, R. J. V. Brissenden, \& J. Barnes (San Francisco, CA: ASP), 173

Turner, M. J. L., Abbey, A., Arnaud, M., et al. 2001, A\&A, 365, L27

Ulmer, A., Fenimore, E. E., Epstein, R. I., et al. 1993, ApJ, 418, 395

van Kerkwijk, M. H., \& Kaplan, D. L. 2008, ApJ, 673, L163

Vasisht, G., \& Gotthelf, E. V. 1997, ApJ, 486, L129

Vaughan, B. A., van der Klis, M., Wood, K. S., et al. 1994, ApJ, 435, 362

Yan, M., Sadeghpour, H. R., \& Dalgarno, A. 1998, ApJ, 496, 1044 